\title{
Maternal dietary fatty acid intake during pregnancy and the risk of preclinical and clinical type 1 diabetes in the offspring
}

\author{
Sari Niinistö ${ }^{1,2 *}$, Hanna-Mari Takkinen ${ }^{3}$, Liisa Uusitalo ${ }^{1}$, Jenna Rautanen ${ }^{1}$, Jaakko Nevalainen ${ }^{4}$, \\ Michael G. Kenward ${ }^{5}$, Mirka Lumia ${ }^{1,3}$, Olli Simell ${ }^{6}$, Riitta Veijola ${ }^{7}$, Jorma Ilonen ${ }^{8,9}$, Mikael Knip ${ }^{10,11,12}$ \\ and Suvi M. Virtanen ${ }^{1,3,13}$ \\ ${ }^{1}$ Nutrition Unit, Department of Lifestyle and Participation, National Institute for Health and Welfare, PO Box 30 , \\ FI-O0271 Helsinki, Finland \\ ${ }^{2}$ Hjelt Institute, University of Helsinki, Helsinki, Finland \\ ${ }^{3}$ The School of Health Sciences, University of Tampere, Tampere, Finland \\ ${ }^{4}$ Statistics/Department of Social Research, University of Turku, Turku, Finland \\ ${ }^{5}$ Medical Statistic Unit, Department of Epidemiology and Population Health, London School of Hygiene \\ and Tropical Medicine, London, UK \\ ${ }^{6}$ Department of Pediatrics, University of Turku, Turku, Finland \\ ${ }^{7}$ Department of Pediatrics, University of Oulu, Oulu, Finland \\ ${ }^{8}$ Immunogenetics Laboratory, University of Turku, Turku, Finland \\ ${ }^{9}$ Department of Clinical Microbiology, University of Eastern Finland, Kuopio, Finland \\ ${ }^{10}$ Children's Hospital, Helsinki University Central Hospital, University of Helsinki, Helsinki, Finland \\ ${ }^{11}$ Folkhälsan Research Center, University of Helsinki, Helsinki, Finland \\ ${ }^{12}$ Department of Pediatrics, Tampere University Hospital, Tampere, Finland \\ ${ }^{13}$ The Science Centre, University of Tampere, Tampere, Finland \\ (Submitted 3 February 2012 - Final revision received 8 August 2013 - Accepted 9 August 2013 - First published online 2 October 2013)
}

\section{Abstract}

The aim of the present study was to examine the associations between the maternal intake of fatty acids during pregnancy and the risk of preclinical and clinical type 1 diabetes in the offspring. The study included 4887 children with human leucocyte antigen (HLA)-conferred type 1 diabetes susceptibility born during the years 1997-2004 from the Finnish Type 1 Diabetes Prediction and Prevention Study. Maternal diet was assessed with a validated FFQ. The offspring were observed at 3- to 12-month intervals for the appearance of type 1 diabetesassociated autoantibodies and development of clinical type 1 diabetes (average follow-up period: 4.6 years (range 0.5-11.5 years)) Altogether, 240 children developed preclinical type 1 diabetes and 112 children developed clinical type 1 diabetes. Piecewise linear log-hazard survival model and Cox proportional-hazards regression were used for statistical analyses. The maternal intake of palmitic acid (hazard ratio (HR) $0.82,95 \%$ CI $0.67,0.99$ ) and high consumption of cheese during pregnancy (highest quarter $v$. intermediate half HR $0.52,95 \%$ CI $0.31,0.87)$ were associated with a decreased risk of clinical type 1 diabetes. The consumption of sour milk products (HR 1.14, $95 \%$ CI 1.02, 1.28), intake of protein from sour milk (HR 1·15, 95\% CI 1.02, 1.29) and intake of fat from fresh milk (HR 1.43, $95 \%$ CI 1.04, 1.96) were associated with an increased risk of preclinical type 1 diabetes, and the intake of low-fat margarines (HR $0.67,95 \%$ CI $0 \cdot 49,0.92)$ was associated with a decreased risk. No conclusive associations between maternal fatty acid intake or food consumption during pregnancy and the development of type 1 diabetes in the offspring were detected.

\section{Key words: Pregnancy: Fatty acids: Type 1 diabetes: Foods: Children: Cohort studies}

Finland has the world's highest, and still increasing, incidence of type 1 diabetes, with the increase being particularly conspicuous among children aged below 5 years ${ }^{(1)}$. Type 1 diabetes develops as the result of an immune-mediated inflammatory reaction that destroys $\beta$-cells in the pancreatic islets $^{(2)}$. Available data suggest that type 1 diabetes is caused by a complex interplay between intestinal microbiota, gut permeability and mucosal immunity ${ }^{(2)}$. Prenatal modulation of the gut is dependent on which factors the fetus encounters via the placenta ${ }^{(3)}$. Accordingly, maternal nutrition during

Abbreviations: DIPP, The Finnish Type 1 Diabetes Prediction and Prevention Study; E\%, percentage of total energy intake; ICA, islet cell antibodies. 
pregnancy may modulate the development of the gut immune system in the offspring. Maternal fatty acid status during pregnancy influences the fatty acid status of the fetus and newborn infant ${ }^{(4)}$. The fatty acids may influence immune programming in many ways, e.g. by altering the lipid composition of fetal cell membranes, cellular metabolism, eicosanoid synthesis and gene expression regulation ${ }^{(4)}$.

Epidemiological studies have suggested that the consumption of $n$-3 fatty acid-rich cod-liver oil during pregnancy or infancy might help in the prevention of the development of type 1 diabetes $^{(5,6)}$. In contrast, in a US cohort, maternal $n-3$ and $n-6$ fatty acid intake has been shown to be not associated with the appearance of islet autoimmunity in the offspring ${ }^{(7)}$, and in a Norwegian cohort study, maternal serum concentrations of $n-3$ fatty acids during pregnancy have been reported to be not associated with the risk of clinical type 1 diabetes in the offspring $^{(8)}$. By contrast, in a US cohort, the intake of $n$ - 3 fatty acids and their content in erythrocyte membranes in children have been shown to be inversely associated with $\beta$-cell autoimmunity ${ }^{(9)}$. Animal studies have also indicated a protective effect of $n-3$ fatty acids $(10,11)$

There are only a few studies that have considered the effect of various fatty acids on the development of type 1 diabetes. In our previous nested case-control analysis in children, serum fatty acid biomarkers of milk consumption have been shown to be positively associated, and linoleic acid inversely associated, with the risk of advanced $\beta$-cell autoimmunity at or close to the time of seroconversion ${ }^{(12)}$. In our recent study in children, we have found that the intake of fat from all milk products is associated with a higher risk of preclinical type 1 diabetes $^{(13)}$. Lipid-induced impairment of $\beta$-cell function (lipotoxicity) has been well established both in vitro and in vivo in animal models in the pathogenesis of type 2 diabetes $^{(14)}$. This raises the question of whether lipotoxicity is involved in the pathogenesis of type 1 diabetes as well. By contrast, MUFA have been shown to protect $\beta$-cells in some in vitro studies ${ }^{(15)}$.

In the present study, we evaluated the intake of fatty acids and foods important in relation to fatty acid intake among pregnant Finnish women and assessed associations with the development of preclinical and clinical type 1 diabetes in their offspring.

\section{Methods}

\section{Participants}

The present investigation is part of the Finnish Type 1 Diabetes Prediction and Prevention (DIPP) Study, which is a large prospective, population-based birth cohort study of Finnish children at an increased genetic risk of type 1 diabetes. Increased human leucocyte antigen (HLA)-conferred disease susceptibility is defined by a high-risk genotype ( $H L A-D Q B 1$ $\left.{ }^{*} 02 /{ }^{*} 0302\right)$ or a moderate-risk genotype (HLA-DQB1*0302/x, where $x=$ other than ${ }^{*} 02,{ }^{*} 0301$ or *0602). Details of the genetic screening methods and enrolment criteria have been published previously ${ }^{(16)}$. The present study was conducted according to the guidelines laid down in the Declaration of Helsinki, and all procedures involving human subjects were approved by the Ethical Committees of the University Hospitals of Oulu and Tampere, Finland. Written informed consent was obtained from all the subjects.

The study population comprised 5605 children with HLAconferred susceptibility to type 1 diabetes. They were born in Tampere or Oulu University Hospital between October 1997 and beginning of September 2004 ( $72 \%$ of those invited). FFQ were completed by the mothers of 4887 children during pregnancy $(87 \%)$.

\section{Assessment of maternal nutrition}

The participants completed a validated 181-item semi-quantitative FFQ concerning their habitual diet during 1 month. The mothers were asked about their food consumption during the eighth month of pregnancy (the month preceding maternity leave in Finland). If more than ten of the 181 food frequency questions were not answered, the FFQ was excluded ( $n$ 53). The FFQ contained questions about the frequency (number of times per d, week or month) and amount of foods consumed, in units of common serving sizes. In addition, the FFQ contained questions related to what type of fat was used in cooking and baking and in salad dressings as well as questions related to the extent of home baking. The individual type and quantity of fat were taken into account when calculating food and nutrient intakes. FFQ content, validity and data processing have been described earlier ${ }^{(17)}$. Information on the intake of fatty acids from all kinds of supplements throughout pregnancy was requested. Sociodemographic and other factors, including maternal education and diabetes in close relatives, were recorded using a structured questionnaire completed by the parents after delivery.

\section{Autoantibody assays and definition of outcome}

The DIPP children were monitored for the appearance of signs of $\beta$-cell autoimmunity by analysing primarily islet cell antibodies (ICA). If a child tested positive for ICA, all his or her samples obtained from birth were analysed for insulin autoantibodies, autoantibodies to $65 \mathrm{kDa}$ isoform of glutamic acid decarboxylase and antibodies to tyrosine phosphataserelated islet antigen 2. Autoantibody samples were obtained during each of the study centre visits, which were scheduled to take place at the age of 3, 6, 12, 18 and 24 months and subsequently at an interval of 12 months. If the child became positive for ICA, the interval between visits was shortened to 3 months. The autoantibodies were analysed in the Research Laboratory, Department of Pediatrics, University of Oulu. ICA, insulin autoantibodies, autoantibodies to $65 \mathrm{kDa}$ isoform of glutamic acid decarboxylase and autoantibodies to tyrosine phosphatase-related islet antigen 2 levels were quantified as described previously ${ }^{(18)}$.

We defined preclinical type 1 diabetes as being repeatedly positive for ICA and one or more of the three other autoantibodies analysed. Altogether, 283 of the 5605 (5.0\%) children seroconverted to repeated ICA positivity and positivity to one of the three other autoantibodies during this period at 
a mean age of 3.6 (range $0 \cdot 5-10 \cdot 0$ ) years. Of the children, 145 ( $2.6 \%)$ had progressed to clinical type 1 diabetes at a mean age of 4.6 (range $0.5-11.5$ ) years. Among the children who progressed to clinical type 1 diabetes, 103 had been repeatedly positive for ICA and at least one other autoantibody. However, fifteen of the remaining forty-two children who progressed to type 1 diabetes had or had had one or more autoantibodies before or at the time of diagnosis. Moreover, ten children who developed type 1 diabetes had been persistently seronegative, with their last blood sample being drawn at a mean age of 4.0 (range $0 \cdot 3-7 \cdot 7$ ) years before diagnosis. A total of seventeen children were not subjected to any autoantibody analysis before the diagnosis of diabetes. Thus, preclinical type 1 diabetes endpoint was defined as the first occurrence of either (1) repeated positivity for ICA in combination with positivity for one or more of the autoantibodies analysed or (2) clinical type 1 diabetes. Due to missing maternal FFQ data, statistical analyses were carried out with 240 preclinical and 112 clinical type 1 diabetes endpoints.

\section{Statistical analyses}

The endpoint of preclinical type 1 diabetes is interval censored and possibly dependent on siblings. To accommodate this structure, a piecewise linear log-hazard survival model was used to analyse the associations of fatty acid intake and food consumption with the risk of preclinical type 1 diabetes, assuming linear log-hazards in the intervals $0-1.99,2-3.99$ and $>4$ years. The results were not sensitive to the particular choice of intervals used. Observation intervals beyond positivity did not contribute to the analyses. The models were fitted using maximum likelihood in SAS PROC NLMIXED, with standard errors of the estimates derived from the observed information matrix. Random effects for family were introduced to accommodate familial dependence, and these were assumed to follow a normal distribution. The proportionality of the hazards was tested by adding linear interaction terms of the exposure variables with time to the models. To illustrate such time-varying effects, the endpoint analyses were carried out in two settings. First, all the data were used in the analyses. Second, data that included endpoint information only up to 3 years of age were used. There was no interval censoring in the diagnosis of clinical type 1 diabetes. Therefore, the associations of fatty acid intake and food consumption with the risk of clinical disease were analysed by Cox proportional-hazards regression.

Fatty acid variables were adjusted for energy intake by the residual method ${ }^{(19)}$ after logarithmic transformation. Food variables were adjusted for energy intake by adding energy intake as a covariate to the survival model. Fatty acid and food variables were used as both continuous and categorical (the latter defined by falling into intervals given by the first and the third sample quartile) explanatory variables in the analyses. Some food variables were dichotomised because of a high proportion of non-users. If there is no indication of a non-linear association between dietary variables and endpoint, only results for continuous covariates are presented. Otherwise, results for categorical variables are presented. In addition to energy-adjusted fatty acid variables, analyses were carried out for the relative proportions of fatty acids (the percentages of total fatty acid intake). We also separately analysed the intakes of fat and protein from milk products.

The possible confounding by background characteristics (hospital of birth, familial diabetes and maternal vocational education) was controlled by adding background variables as covariates to the survival model; SAS version 9.2 (SAS Institute) was used in the analyses. Statistical significance was taken as less than $5 \%$.

\section{Results}

The characteristics of the study children are given in Table 1 . The maternal average daily intake of fatty acids from foods and mean consumption of foods during pregnancy are given in Tables 2 and 3, respectively. The number of users of supplements containing fatty acids was small ( $n$ 81, $1.7 \%$ of all

Table 1. Characteristics of the study participants

(Number of participants and percentages)

\begin{tabular}{|c|c|c|c|c|c|c|}
\hline \multirow[b]{2}{*}{ Characteristics } & \multicolumn{2}{|r|}{ Distribution } & \multicolumn{2}{|c|}{$\begin{array}{l}\text { With endpoint preclinical } \\
\text { type } 1 \text { diabetes }\end{array}$} & \multicolumn{2}{|c|}{$\begin{array}{c}\text { With endpoint type } 1 \\
\text { diabetes }\end{array}$} \\
\hline & $n$ & Percentage of total & $n$ & Percentage of total & $n$ & Percentage of total \\
\hline Total $n$ & 4887 & 100 & 240 & 4.9 & 112 & $2 \cdot 3$ \\
\hline \multicolumn{7}{|l|}{ Genetic risk group } \\
\hline High risk $\left(D Q B 1^{*} 02 /{ }^{*} 0302\right)$ & 961 & $19 \cdot 7$ & 77 & $8 \cdot 0$ & 39 & $4 \cdot 1$ \\
\hline Moderate risk $\left(D Q B 1{ }^{*} 0302 / x^{*}\right)$ & 3926 & $80 \cdot 3$ & 163 & $4 \cdot 2$ & 73 & 1.9 \\
\hline \multicolumn{7}{|l|}{ Familial diabetes } \\
\hline Yes & 285 & $5 \cdot 8$ & 32 & $11 \cdot 2$ & 19 & $6 \cdot 7$ \\
\hline No & 4421 & $90 \cdot 5$ & 202 & $4 \cdot 6$ & 91 & $2 \cdot 1$ \\
\hline Missing data & 181 & 3.7 & 6 & $3 \cdot 3$ & 2 & $1 \cdot 1$ \\
\hline \multicolumn{7}{|l|}{ Maternal education } \\
\hline None & 297 & $6 \cdot 1$ & 27 & $9 \cdot 1$ & 11 & $3 \cdot 7$ \\
\hline Vocational school/training & 1292 & $26 \cdot 4$ & 58 & 4.5 & 22 & 1.7 \\
\hline Upper secondary/vocational & 2067 & $42 \cdot 3$ & 88 & $4 \cdot 3$ & 46 & $2 \cdot 2$ \\
\hline Academic & 1103 & $22 \cdot 6$ & 60 & 5.4 & 29 & $2 \cdot 6$ \\
\hline Missing data & 128 & $2 \cdot 6$ & 7 & $5 \cdot 5$ & 4 & $3 \cdot 1$ \\
\hline
\end{tabular}

${ }^{*} x$ not equal to ${ }^{*} 02,{ }^{*} 0301$ and ${ }^{*} 0602$. 


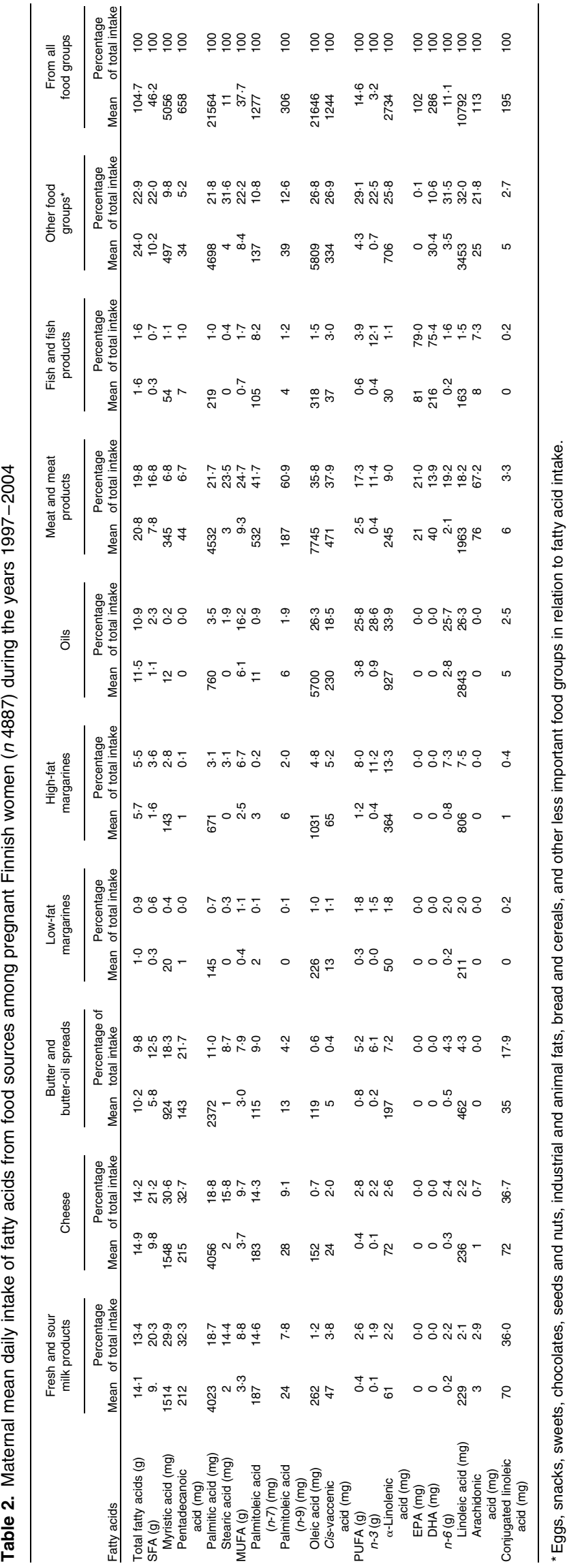

women). Thus, intakes of fatty acids only from food sources are presented and used in the aetiological analysis. The maternal mean daily intake of total fatty acids was $32.8 \%$ of energy intake (E\%), SFA $14.4 \mathrm{E} \%$, MUFA $11.7 \mathrm{E} \%$, PUFA $4.7 \mathrm{E} \%, n-3$ PUFA $1.0 \mathrm{E} \%$ and $n-6$ PUFA $3.5 \mathrm{E} \%$. Fresh and sour milk, cheese, butter and butter-oil spreads were the most important sources of SFA, the most important of which were myristic, pentadecanoic and palmitic acids (Table 2). Red meat and meat products were the main sources of palmitoleic acid (both $n-9$ and $n-7)$, stearic acid, oleic acid, cis-vaccenic acid and arachidonic acid. Linoleic acid and $\alpha$-linolenic acid were derived mainly from oils and EPA and DHA from fish.

The maternal intake of saturated palmitic acid (HR 0.82, $95 \%$ CI $0.67,0.99 ; P=0.039)$ was weakly associated with a decreased risk of clinical type 1 diabetes (Table 4). For the endpoint clinical type 1 diabetes, interactions between fatty acids and time were significant for the following fatty acids: palmitoleic acid isomers $16: 1 n-7 \quad(P=0.019)$ and $16: 1 n-9(P=0.014)$; EPA $(P=0 \cdot 037)$; dihomo- $\gamma$-linolenic acid $(P=0 \cdot 013)$. To illustrate these apparently time-varying effects, analyses were carried out separately for children aged below 3 years. When the follow-up time was less than 3 years, monounsaturated palmitoleic acid (16:1n-7) (HR 0.54, $95 \%$ CI 0.36, $0 \cdot 86 ; P=0.014)$ was associated with a decreased risk of clinical type 1 diabetes, but not during the overall follow-up period.

High consumption of cheese was associated with a decreased risk of clinical type 1 diabetes (highest quarter $v$. intermediate half HR $0.52(95 \% \mathrm{CI} \mathrm{0.31,} \mathrm{0.87))} \mathrm{(Table} \mathrm{3).} \mathrm{The} \mathrm{consumption}$ of sour milk was associated with an increased risk of preclinical type 1 diabetes (HR 1·14, $95 \%$ CI 1.02, 1.28), and the consumption of low-fat margarines was associated with a decreased risk (HR 0.67, 95\% CI 0.49, 0.92). No significant interactions with time were detected for any of the foods, and results are thus presented for only the whole period. The adjustment for the putative confounding variables (genetic risk, hospital of birth, familial diabetes and maternal vocational education) modified the results slightly (Tables 3, 4 and 5).

To determine which fraction of milk products accounts for the observed associations with the development of preclinical or clinical type 1 diabetes, we separately examined the intakes of fat and protein from milk products. High intakes of fat from fresh milk (HR 1.43,95\% CI 1.04, 1.96) and protein from sour milk (HR 1.15, 95\% CI 1.02, 1.29) were associated with an increased risk of preclinical type 1 diabetes (Table 5).

\section{Discussion}

In the present prospective cohort study with genetically susceptible offspring, it was found that maternal diet during pregnancy was weakly associated with the development of preclinical and clinical type 1 diabetes. The maternal intake of palmitic acid and consumption of cheese during pregnancy were inversely associated with the development of clinical type 1 diabetes. The consumption of sour milk products, intake of protein from sour milk and intake of fat from fresh milk products were associated with an increased risk of preclinical type 1 diabetes, and the intake of low-fat margarines was associated with a decreased risk. 
Table 3. Associations of maternal food consumption during pregnancy with the risk of preclinical and clinical type 1 diabetes in the offspring* (Mean values and standard deviations; hazard ratios (HR) and $95 \%$ confidence intervals)

\begin{tabular}{|c|c|c|c|c|c|c|c|c|c|c|c|c|c|c|}
\hline \multirow[b]{2}{*}{$\begin{array}{l}\text { Food group } \\
\text { (g/d per times per week) }\end{array}$} & \multirow[b]{2}{*}{ Mean } & \multirow[b]{2}{*}{ SD } & \multicolumn{6}{|c|}{ Preclinical type 1 diabetes ( $n$ 4887, $n 240$ ) } & \multicolumn{6}{|c|}{ Type 1 diabetes ( $n$ 4887, $n 112)$} \\
\hline & & & $\mathrm{HR} \dagger$ & $95 \% \mathrm{Cl}$ & $P \ddagger$ & $\begin{array}{l}\text { Adjusted } \\
\text { HR§ }\end{array}$ & $95 \% \mathrm{Cl}$ & $P \ddagger$ & $\mathrm{HR} \dagger$ & $95 \% \mathrm{Cl}$ & $P \ddagger$ & $\begin{array}{l}\text { Adjusted } \\
\text { HR§ }\end{array}$ & $95 \% \mathrm{Cl}$ & $P \ddagger$ \\
\hline $\begin{array}{l}\text { Cows' milk products } \\
\text { (continuous) (g) }\end{array}$ & 856 & 422 & 1.05 & $0.91,1.21$ & 0.521 & 1.02 & $0.88,1 \cdot 20$ & 0.754 & 1.01 & $0.81,1.25$ & 0.943 & 1.01 & $0.81,1 \cdot 13$ & 0.921 \\
\hline Fresh milk (g) & 526 & 369 & & & & & & & & & & & & \\
\hline Lowest quarter: $<213$ & & & 0.94 & $0.69,1.28$ & & 0.94 & $0.68,1.30$ & & $0 \cdot 87$ & $0.56,1.36$ & & 0.88 & $0.56,1.38$ & \\
\hline Intermediate half & & & 1 & & 0.421 & 1 & & 0.196 & 1 & & 0.071 & 1 & & 0.037 \\
\hline Highest quarter: $>760$ & & & 0.81 & $0.58,1.12$ & & 0.73 & $0.51,1.04$ & & 0.56 & $0.33,0.94$ & & 0.51 & $0.30,0.88$ & \\
\hline Cheese (g) & 65 & 46 & & & & & & & & & & & & \\
\hline Lowest quarter: $<31$ & & & 0.82 & $0.60,1 \cdot 13$ & & 0.78 & $0.56,1.09$ & & 0.65 & $0.40,1.04$ & & 0.61 & $0.38,1.00$ & \\
\hline Intermediate half & & & 1 & & $0 \cdot 100$ & 1 & & 0.127 & 1 & & 0.015 & 1 & & 0.023 \\
\hline Highest quarter: > 86 & & & 0.71 & $0.51,0.99$ & & 0.74 & $0.52,1.04$ & & 0.52 & $0.31,0.87$ & & 0.55 & $0.33,0.94$ & \\
\hline Sour milk (continuous) (g) & 231 & 199 & $1 \cdot 14$ & $1 \cdot 02,1 \cdot 28$ & 0.030 & $1 \cdot 16$ & $1.03,1.30$ & 0.024 & $1 \cdot 14$ & $0.96,1.35$ & 0.155 & $1 \cdot 15$ & $0.97,1.37$ & 0.397 \\
\hline $\begin{array}{l}\text { Butter and butter-oil spreads } \\
\text { (continuous) (g) }\end{array}$ & 15 & 15 & 0.92 & $0.80,1.07$ & 0.293 & 0.96 & $0.83,1.11$ & 0.540 & 0.90 & $0.73,1.12$ & 0.330 & 0.92 & $0.74,1.14$ & 0.421 \\
\hline Low-fat margarines (g) & 2.5 & 6 & & & & & & & & & & & & \\
\hline $\begin{array}{l}\text { Users } v \text {. non-users } \\
\text { High-fat margarines }(\mathrm{g})\end{array}$ & & & 0.67 & $0.49,0.92$ & 0.010 & 0.66 & $0.48,0.91$ & 0.009 & 0.70 & $0.44,1 \cdot 10$ & 0.104 & 0.66 & $0.41,1.05$ & 0.066 \\
\hline $\begin{array}{l}\text { High-fat margarines (g) } \\
\text { Users v. non-users }\end{array}$ & 7 & 13 & & & & & & & & & & & & \\
\hline Users $v$. non-users & & & $1 \cdot 12$ & $1.00,1 \cdot 26$ & 0.378 & $1 \cdot 12$ & $0.85,1.46$ & 0.422 & $1 \cdot 16$ & $0.80,1.69$ & 0.431 & $1 \cdot 18$ & $0.81,1.73$ & 0.391 \\
\hline Oil (continuous) (g) & 11 & 7 & 0.96 & $0.84,1 \cdot 11$ & 0.677 & 0.96 & $0.82,1 \cdot 11$ & 0.557 & 0.96 & $0.78,1 \cdot 17$ & 0.661 & 1.03 & $0.83,1.28$ & 0.804 \\
\hline $\begin{array}{l}\text { Red meat and meat products } \\
\text { (continuous) (g) }\end{array}$ & 127 & 61 & $1 \cdot 10$ & $0.94,1.28$ & 0.231 & 1.09 & $0.93,1.27$ & 0.308 & $1 \cdot 15$ & $0.92,1.44$ & 0.213 & $1 \cdot 16$ & $0.92,1.45$ & 0.208 \\
\hline Poultry (continuous) (g) & 37 & 32 & 0.97 & $0.85,1 \cdot 12$ & 0.718 & 1.02 & $0.89,1 \cdot 17$ & 0.805 & 0.98 & $0.79,1.20$ & 0.831 & 1.03 & $0.83,1.27$ & 0.810 \\
\hline $\begin{array}{l}\text { Fatty fish\| (continuous) } \\
\text { (times per week) }\end{array}$ & $1 \cdot 3$ & 1.3 & 0.89 & $0.76,1.03$ & $0 \cdot 108$ & 0.91 & $0.78,1.06$ & $0 \cdot 193$ & 0.92 & $0.74,1.14$ & 0.414 & 0.91 & $0.73,1.14$ & 0.411 \\
\hline $\begin{array}{l}\text { Lean fish } \text { (continuous) } \\
\text { (times per week) }\end{array}$ & 0.7 & 0.7 & 1.00 & $0.88,1.14$ & 0.966 & 0.97 & $0.85,1 \cdot 12$ & 0.687 & 1.05 & $0 \cdot 88-1 \cdot 26$ & 0.604 & 0.99 & $0.81,1.20$ & 0.883 \\
\hline
\end{tabular}

* For continuous food variables, HR describes the change in risk, when the food variable is changed by an amount corresponding to its standard deviation. For categorical food variables, reference category is the intermediate half, and for dichotomised variables, reference category is the non-users.

† Model was adjusted for maternal energy intake.

fLikelihood ratio test was used to test whether the model with and without the food variables differed.

Sodel was adjusted for mateational education.

$\|$ Salmon, rainbow trout, Baltic herring, mackerel, lavaret, trout, European cisco, pike-perch, flounder, bream, rosefish, herring, anchovy and tuna.
ๆ Fish fingers, frozen fish, perch, northern pike, burbot, shrimps and shell. 
Table 4. Associations of maternal fatty acid intake during pregnancy with the risk of preclinical and clinical type 1 diabetes in the offspring ${ }^{\star}$ (Hazard ratios (HR) and $95 \%$ confidence intervals)

\begin{tabular}{|c|c|c|c|c|c|c|c|c|c|c|c|c|}
\hline \multirow[b]{3}{*}{ Fatty acids (g/mg) } & \multicolumn{6}{|c|}{ Preclinical type 1 diabetes } & \multicolumn{6}{|c|}{ Type 1 diabetes } \\
\hline & \multicolumn{3}{|c|}{ All $(n 4887, n 240)$} & \multicolumn{3}{|c|}{ All $(n 4887, n 240)$} & \multicolumn{3}{|c|}{ All $(n 4887, n 112)$} & \multicolumn{3}{|c|}{ All $(n 4887, n 112)$} \\
\hline & $\mathrm{HR}$ & $95 \% \mathrm{Cl}$ & $P \dagger$ & Adjusted HR $\ddagger$ & $95 \% \mathrm{Cl}$ & $P \dagger$ & $\mathrm{HR}$ & $95 \% \mathrm{Cl}$ & P† & Adjusted HR $\ddagger$ & $95 \% \mathrm{Cl}$ & $P \dagger$ \\
\hline Total fatty acids (g) & 0.92 & $0.81,1.05$ & 0.247 & 0.92 & $0.80,1.05$ & 0.224 & 0.86 & $0.71,1.05$ & 0.134 & 0.86 & $0.71,1.05$ & 0.143 \\
\hline SFA (g) & 0.90 & $0.79,1.03$ & 0.127 & 0.91 & $0.79,1.04$ & 0.163 & 0.83 & $0.68,1.01$ & 0.056 & 0.84 & $0.69,1.03$ & 0.087 \\
\hline Myristic acid (14:0) (mg) & 0.90 & $0.79,1.03$ & 0.133 & 0.92 & $0.81,1.06$ & 0.246 & 0.82 & $0.67,1.00$ & 0.041 & 0.86 & $0.71,1.05$ & 0.132 \\
\hline Pentadecanoic acid $(15: 0)(\mathrm{mg})$ & 0.91 & $0.80,1.04$ & 0.172 & 0.93 & $0.81,1.06$ & 0.172 & 0.84 & $0.69,1.03$ & 0.083 & 0.87 & $0.71,1.06$ & 0.153 \\
\hline Palmitic acid $(16: 0)(\mathrm{mg})$ & 0.89 & $0.79,1.02$ & 0.099 & 0.90 & $0.79,1.03$ & 0.134 & 0.82 & $0.67,0.99$ & 0.039 & 0.84 & $0.69,1.02$ & 0.074 \\
\hline Stearic acid $(18: 0)(\mathrm{mg})$ & 0.91 & $0.80,1.03$ & 0.150 & 0.91 & $0.79,1.04$ & 0.162 & 0.84 & $0.69,1.03$ & 0.085 & 0.85 & $0.70,1.04$ & 0.120 \\
\hline MUFA (g) & 0.96 & $0.84,1.09$ & 0.551 & 0.96 & $0.82,1.08$ & 0.404 & 0.93 & $0.76,1.12$ & 0.427 & 0.91 & $0.75,1.11$ & 0.348 \\
\hline Palmitoleic acid $(16: 1 n-7)(\mathrm{mg})$ & 0.91 & $0.79,1.03$ & 0.145 & 0.93 & $0.81,1.07$ & 0.307 & 0.90 & $0.74,1.09$ & 0.265 & 0.95 & $0.78,1.15$ & 0.571 \\
\hline Palmitoleic acid $(16: 1 n-9)(\mathrm{mg})$ & 0.96 & $0.84,1 \cdot 10$ & 0.562 & 0.98 & $0.86,1.12$ & 0.753 & 1.02 & $0.84,1.24$ & 0.833 & 1.05 & $0.87,1.26$ & 0.649 \\
\hline Oleic acid $(18: 1 n-9)(\mathrm{mg})$ & 0.93 & $0.82,1.06$ & 0.296 & 1.02 & $0.89,1.16$ & 0.802 & 0.92 & $0.76,1 \cdot 11$ & 0.386 & 0.99 & $0.82,1 \cdot 20$ & 0.926 \\
\hline Cis-vaccenic acid $(18: 1 n-7)(\mathrm{mg})$ & 0.95 & $0.84,1.08$ & 0.456 & $10 \cdot 03$ & $0.84,1.08$ & 0.693 & 0.97 & $0.80,1.16$ & 0.707 & 1.04 & $0.87,1.26$ & 0.660 \\
\hline PUFA (g) & 1.01 & $0.89,1.14$ & 0.887 & 0.96 & $0.83,1.09$ & 0.502 & 1.00 & $0.83,1.20$ & 0.975 & 0.98 & $0.80,1 \cdot 19$ & 0.798 \\
\hline$n-3(\mathrm{~g})$ & 0.97 & $0.86,1 \cdot 10$ & 0.685 & 0.97 & $0.86,1 \cdot 10$ & 0.685 & 1.02 & $0.85,1.23$ & 0.802 & 0.99 & $0.82,1.20$ & 0.917 \\
\hline$\alpha$-Linolenic acid $(18: 3 n-3)(\mathrm{mg})$ & 0.99 & $0.87,1.12$ & 0.856 & 0.97 & $0.85,1 \cdot 10$ & 0.607 & 1.02 & $0.85,1.22$ & 0.848 & 0.98 & $0.81,1.19$ & 0.844 \\
\hline EPA $(20: 5 n-3)(\mathrm{mg})$ & 0.94 & $0.82,1.08$ & 0.401 & 0.96 & $0.83,1 \cdot 11$ & 0.560 & 1.03 & $0.86,1 \cdot 24$ & 0.730 & 1.04 & $0.85,1 \cdot 26$ & 0.719 \\
\hline DHA $(22: 6 n-3)(\mathrm{mg})$ & 0.96 & $0.84,1 \cdot 10$ & 0.531 & 0.97 & $0.84,1 \cdot 12$ & 0.708 & 1.04 & $0.87,1.25$ & 0.669 & 1.04 & $0.86,1.27$ & 0.687 \\
\hline$n-6(\mathrm{~g})$ & 1.02 & $0.90,1.16$ & 0.707 & 1.01 & $0.88,1.15$ & 0.910 & 1.00 & $0.83,1.21$ & 0.996 & 0.98 & $0.80,1.19$ & 0.219 \\
\hline Linoleic acid $(18: 2 n-6)(\mathrm{mg})$ & 1.01 & $0.89,1.15$ & 0.796 & 1.01 & $0.88,1.15$ & 0.942 & 1.00 & $0.83,1.20$ & 0.995 & 1.00 & $0.83,1.20$ & 0.995 \\
\hline Arachidonic acid $(20: 4 n-6)(\mathrm{mg})$ & 1.03 & $0.91,1.17$ & 0.608 & 1.04 & $0.92,1.19$ & 0.537 & 1.08 & $0.90,1.30$ & 0.397 & 1.08 & $0.90,1.30$ & 0.397 \\
\hline$\gamma$-Linolenic acid $(18: 3 n-6)(\mathrm{mg})$ & 0.92 & $0.78,1.09$ & 0.310 & 0.93 & $0.81,1.07$ & 0.293 & 0.84 & $0.65,1.10$ & $0 \cdot 187$ & 0.84 & $0.65,1 \cdot 10$ & $0 \cdot 187$ \\
\hline Dihomo- $\gamma$-linolenic acid $(20: 3 n-6)(\mathrm{mg})$ & 0.96 & $0.84,1.10$ & 0.556 & 0.94 & $0.82,1.08$ & 0.384 & 1.01 & $0.84,1.22$ & 0.923 & 1.02 & $0.84,1.22$ & 0.874 \\
\hline Ratio of $n-6: n-3$ & 1.05 & $0.94,1 \cdot 18$ & 0.407 & 1.06 & $0.94,1.20$ & 0.332 & 0.96 & $0.79,1 \cdot 17$ & 0.698 & 0.98 & $0.81,1 \cdot 19$ & 0.832 \\
\hline Conjugated linoleic acid $(18: 2 n-6)(\mathrm{mg})$ & 0.93 & $0.81,1.06$ & 0.757 & 0.94 & $0.82,1.08$ & 0.377 & 0.88 & $0.72,1.07$ & 0.186 & 0.89 & $0.73,1.08$ & 0.220 \\
\hline
\end{tabular}

* Fatty acid variables used in statistical analyses were energy adjusted using Willett's residual method. HR describes change in risk, when fatty acid variable is changed by an amount corresponding to its standard deviation. These results are not adjusted for any potential confounders.

tLikelihood ratio test was used to test whether the model with and without the fatty acid variables differed.

¥ Model was adjusted for genetic risk, hospital of birth, familial diabetes and maternal vocational education 
Table 5. Associations of maternal intake of fat and protein from dairy products during pregnancy with the risk of preclinical and clinical type 1 diabetes in the offspring* (Hazard ratios (HR) and $95 \%$ confidence intervals)

\begin{tabular}{|c|c|c|c|c|c|c|c|c|c|c|c|c|}
\hline \multirow[b]{2}{*}{ Fat and protein from dairy products (g) } & \multicolumn{6}{|c|}{ Preclinical type 1 diabetes ( $n$ 4887, $n$ 240) } & \multicolumn{6}{|c|}{ Type 1 diabetes ( $n 4887, n 112)$} \\
\hline & $\mathrm{HR} \dagger$ & $95 \% \mathrm{Cl}$ & $P \ddagger$ & Adjusted HR§ & $95 \% \mathrm{Cl}$ & $P \ddagger$ & $\mathrm{HR} \dagger$ & $95 \% \mathrm{Cl}$ & $P \ddagger$ & Adjusted HR§ & $95 \% \mathrm{Cl}$ & $P \ddagger$ \\
\hline \multicolumn{13}{|l|}{ Fat from } \\
\hline All cows' milk products (continuous) & 0.89 & $0.75,1.06$ & 0.197 & 0.91 & $0.76,1.08$ & 0.275 & 0.87 & $0.68,1.12$ & 0.265 & 0.90 & $0.70,1.16$ & 0.408 \\
\hline \multicolumn{13}{|l|}{ Fresh milk } \\
\hline Lowest quarter & 1.35 & $0.98,1.87$ & \multirow{3}{*}{0.046} & 1.31 & $0.95,1.82$ & \multirow{3}{*}{0.081} & 1.62 & $1.04,2.52$ & \multirow{3}{*}{0.114} & 1.64 & $1.04,2.59$ & \multirow{3}{*}{0.112} \\
\hline Intermediate half & 1 & & & 1 & & & 1 & & & 1 & & \\
\hline Highest quarter & 1.43 & $1.04,1.96$ & & 1.39 & $1.01,1.92$ & & $1 \cdot 16$ & $0.72,1.86$ & & 1.17 & $0.72,1.90$ & \\
\hline Cheese (continuous) & 0.86 & $0.74,1.01$ & 0.053 & 0.88 & $0.75,1.03$ & 0.101 & 0.89 & $0.71,1 \cdot 10$ & 0.266 & 0.92 & $0.74,1.15$ & 0.459 \\
\hline Sour milk (continuous) & 1.08 & $0.95,1.22$ & 0.280 & 1.07 & $0.94,1.22$ & 0.310 & 1.06 & $0.88,1.28$ & 0.549 & 1.06 & $0.88,1.27$ & $0.57 \varepsilon$ \\
\hline \multicolumn{13}{|l|}{ Protein from } \\
\hline All cows' milk products (continuous) & 0.94 & $0.80,1.11$ & 0.468 & 0.94 & $0.79,1 \cdot 11$ & 0.480 & 0.92 & $0.73,1.17$ & 0.497 & 0.96 & $0.75,1.22$ & 0.713 \\
\hline \multicolumn{13}{|l|}{ Fresh milk } \\
\hline Lowest quarter & 0.94 & $0.68,1.29$ & \multirow{3}{*}{0.340} & 0.94 & $0.68,1.29$ & \multirow{3}{*}{0.155} & 0.88 & $0.56,1.37$ & \multirow{3}{*}{0.077} & 0.88 & $0.56,1.39$ & \multirow{3}{*}{0.045} \\
\hline Intermediate half & 1 & & & 1 & & & 1 & & & 1 & & \\
\hline Highest quarter & 0.78 & $0.55,1.09$ & & 0.71 & $0.50,1.01$ & & 0.56 & $0.33,0.95$ & & 0.52 & $0.30,0.89$ & \\
\hline \multicolumn{13}{|l|}{ Cheese } \\
\hline Lowest quarter & 0.92 & $0.67,1.27$ & \multirow{3}{*}{0.177} & 0.86 & $0.62,1.19$ & \multirow{3}{*}{0.241} & 0.80 & $0.51,1.27$ & \multirow{3}{*}{0.070} & 0.71 & $0.44,1.15$ & \multirow{3}{*}{0.088} \\
\hline Intermediate half & 1 & & & 1 & & & 1 & & & 1 & & \\
\hline Highest quarter & 0.72 & $0.51,1.02$ & & 0.75 & $0.53,1.07$ & & 0.56 & $0.34,0.94$ & & 0.60 & $0.36,1.01$ & \\
\hline Sour milk (continuous) & 1.15 & $1.02,1.29$ & 0.030 & 1.16 & $1.03,1.31$ & 0.023 & 1.14 & $0.96,1.36$ & 0.147 & 1.16 & $0.98,1.38$ & 0.112 \\
\hline
\end{tabular}

* For continuous fat and protein variables, HR describes change in risk, when the food variable is changed by an amount corresponding to its standard deviation. For categorical fat and protein variables, reference category is the intermediate half.

† Model was adjusted for energy intake.

the model with and without the food variables differed.

$\S$ Model was adjusted for maternal energy intake, genetic risk, hospital of birth, familial diabetes and maternal vocational education. 
The strength of the present study is the population-based prospective cohort setting as well as the fact that the endpoints used comprised both preclinical type 1 diabetes and, separately, clinical type 1 diabetes. The endpoint 'preclinical type 1 diabetes' is strongly associated with clinical disease ${ }^{(20)}$. The FFQ used in the present study was validated against two $5 \mathrm{~d}$ food records, and it estimated the consumption of food groups relatively well ${ }^{(17)}$. The consumption of milk products had very good validity (Pearson's correlation coefficients for different milk products varied between 0.58 and 0.86$)^{(17)}$.

The major limitation of the study is the high number of fatty acids and food groups analysed. Altogether, we tested over seventy associations. None of the associations was highly significant and may be false positive and occur due to chance. Therefore, the results must be interpreted with caution. Another limitation is that the children's diet during infancy was not taken into account, because the food records of all the children were not available. In the validation study, for some important food sources of fat, the correlations measured by FFQ and food records were low, as Pearson's correlation coefficients for the consumption of oils and low-fat spreads were $0 \cdot 22$ and $0 \cdot 25$, respectively ${ }^{(17)}$

The present observation is the first epidemiological one to indicate that the maternal intake of palmitic acid, which reflects the consumption of certain milk products, and consumption of cheese are inversely associated with clinical type 1 diabetes in the offspring. The results indicate that maternal exposure to certain milk products during pregnancy might induce tolerance to cows' milk in genetically susceptible offspring. A previous study has shown that the maternal intake of protein from cheese during pregnancy and maternal intake of protein from fresh milk products and cheese during lactation are marginally inversely associated with the humoral immune responses to cows' milk proteins in offspring with a high risk of type 1 diabetes $^{(21)}$. An enhanced humoral immune response to various cows' milk proteins has been observed in infancy in children who later were diagnosed with type 1 diabetes $^{(22)}$.

In contrast to the inverse associations between the maternal intake of milk-related fatty acids and the risk of type 1 diabetes in the offspring in the present study, our previous casecontrol analysis in 315 children has shown that the fatty acid biomarkers of milk consumption are directly associated with the risk of islet autoimmunity at or before the time of seroconversion $^{(12)}$. Our newest findings in children were that the consumption of fresh milk products and cows' milk-based infant formulas and the intake of fat from all milk products and intake of protein from fresh milk products are weakly associated with a higher risk of advanced preclinical type 1 diabetes $^{(13)}$. These findings support the hypothesis that the direct exposure of children to cows' milk leads to immunisation or the results could be partly explained by possible lipotoxicity. Several other case-control and cohort studies as well as randomised trial findings also support the hypothesis that cows' milk is associated with the development of type 1 diabetes, although there are conflicting results also ${ }^{(23,24)}$. Savilahti \& Saarinen ${ }^{(25)}$ observed that very early exposure to cows' milk in the maternity hospital may decrease the risk of type 1 diabetes in the offspring by the age of 8 years.
In the present study, the maternal intake of palmitoleic acid (16:1n-7) during pregnancy was inversely associated with the risk of clinical type 1 diabetes when the children were observed up to the age of 3 years. An inverse association of palmitoleic acid intake may reflect the maternal consumption of meat or dairy products. The observation could also reflect that the effect of palmitoleic acid may be protective in accordance with some in vitro studies ${ }^{(15)}$.

Type 1 diabetes has been shown to be associated with increased inflammation, and $n-3$ fatty acids have been implicated as anti-inflammatory agents, while $n$ - 6 acids fatty acids have been shown to be pro-inflammatory agents ${ }^{(26)}$. In line with other cohort findings, the maternal intake of $n-3$ or $n-6$ fatty acids during pregnancy was not associated with the risk of preclinical or clinical type 1 diabetes in the present study ${ }^{(7,8)}$.

In accordance with our previous analyses using a smaller dataset, the maternal intake of low-fat margarines during pregnancy was found to be associated with a decreased risk of preclinical type 1 diabetes $^{(27)}$. In contrast to our earlier results ${ }^{(27)}$, butter was not associated with the risk of prediabetes and sour milk was associated with an increased risk of prediabetes in the present study. However, in the present study, the maternal intake of protein from sour milk was also associated with an increased risk of prediabetes.

The present findings that the maternal intake of fat from fresh milk products is weakly associated with an increased risk of advanced preclinical type 1 diabetes may indicate lipotoxicity to be responsible for the pathogenesis of type 1 diabetes. However, palmitic acid intake exhibited a protective association with the clinical type 1 diabetes endpoint.

In other analyses carried out in the cohort of the present study, interesting findings emerged for fatty acids and other immune-mediated endpoints, and these suggest that maternal diet and fatty acid intake during pregnancy may influence the development of the immune system in the offspring. High maternal consumption of butter and a high ratio of $n-6: n-3$ fatty acids during pregnancy have been reported to be associated with an increased risk of allergic rhinitis in the offspring $^{(28)}$. The maternal intakes of $\alpha$-linoleic acid ( $\left.n-3\right)$, total $n-3$ PUFA, SFA and palmitic acid during pregnancy have been reported to be associated with a decreased risk of asthma and that of arachidonic acid ( $n-6)$ with an increased risk $^{(29)}$. These and present findings support the importance of fatty acids when studying relationships between nutrition and immune-mediated diseases.

\section{Acknowledgements}

The authors express their gratitude to the children and parents who participated in the present study. They thank the DIPP research nurses, doctors, nutritionists and laboratory staff for their excellent collaboration over the years. They are also grateful to Heli Tapanainen for her skilful assistance. An abstract of the present study has been published at the 46th Annual meeting of the European Diabetes Epidemiology Group, 15-18 May 2011.

The present study was supported by the Doctoral Program in Public Health, the Academy of Finland (grants 63672, 79685, 
79686, 80846, 201988 and 210632), the Finnish Diabetes Association, the Finnish Diabetes Research Foundation, the Finnish Pediatric Research Foundation, the Häme Foundation of the Finnish Culture Fund, the Juho Vainio Foundation, the Yrjö Jahnsson Foundation, Medical Research Funds, Turku, Oulu and Tampere University Hospitals' Juvenile Diabetes Research Foundation (grants 197032, 4-1998-274, 4-1999-731 and 4-2001-435), the Novo Nordisk Foundation and the EU Biomed 2 Program (BMH4-CT98-3314). None of the funders had any role in the design and analysis of the study or in the writing of this article.

The authors' contributions were as follows: S. N. shared responsibility for the writing of the manuscript with S. M. V., H.-M. T., L. U., M. K., J. N. and M. L.; S. M. V., L. U., S. N., H.-M. T. and J. N. were responsible for the study concept and design; J. R. updated the fatty acid values of their national database; J. I., M. K. and O. S. designed the DIPP Study; S. M. V. designed the nutrition study within the DIPP Study; H.-M. T. carried out the statistical analyses under the supervision of J. N. and M. G. K.; R. V. had responsibility for the clinical work in Oulu. All the authors contributed to the critical revision of the manuscript.

The authors declare that there is no conflict of interest associated with this paper.

\section{References}

1. Harjutsalo V, Sjoberg L \& Tuomilehto J (2008) Time trends in the incidence of type 1 diabetes in Finnish children: a cohort study. Lancet 371, 1777-1782.

2. Vaarala O, Atkinson MA \& Neu J (2008) The "perfect storm" for type 1 diabetes: the complex interplay between intestinal microbiota, gut permeability, and mucosal immunity. Diabetes 57, 2555-2562.

3. Blumer N, Pfefferle PI \& Renz H (2007) Development of mucosal immune function in the intrauterine and early postnatal environment. Curr Opin Gastroenterol 23, 655-660.

4. Prescott SL \& Dunstan JA (2007) Prenatal fatty acid status and immune development: the pathways and the evidence. Lipids 42, 801-810.

5. Stene LC \& Joner G (2003) Norwegian Childhood Diabetes Study Group Use of cod liver oil during the first year of life is associated with lower risk of childhood-onset type 1 diabetes: a large, population-based, case-control study. Am J Clin Nutr 78, 1128-1134.

6. Stene LC, Ulriksen J, Magnus P, et al. (2000) Use of cod liver oil during pregnancy associated with lower risk of type I diabetes in the offspring. Diabetologia 43, 1093-1098.

7. Fronczak CM, Baron AE, Chase HP, et al. (2003) In utero dietary exposures and risk of islet autoimmunity in children. Diabetes Care 26, 3237-3242.

8. Sorensen IM, Joner G, Jenum PA, et al. (2012) Maternal fatty acid status during pregnancy and risk of type 1 diabetes in the offspring. Diabetes Metab Res Rev 28, 431-438.

9. Norris JM, Yin X, Lamb MM, et al. (2007) Omega-3 polyunsaturated fatty acid intake and islet autoimmunity in children at increased risk for type 1 diabetes. JAMA 298, 1420-1428.

10. Kagohashi Y, Abiru N, Kobayashi M, et al. (2010) Maternal dietary $n-6 / n-3$ fatty acid ratio affects type 1 diabetes development in the offspring of non-obese diabetic mice. Congenit Anom (Kyoto) 50, 212-220.
11. Wei D, LiJ, Shen M, et al. (2010) Cellular production of $n$-3 PUFAs and reduction of $n-6$-to- $n-3$ ratios in the pancreatic beta-cells and islets enhance insulin secretion and confer protection against cytokine-induced cell death. Diabetes 59, 471-478.

12. Virtanen SM, Niinisto S, Nevalainen J, et al. (2010) Serum fatty acids and risk of advanced beta-cell autoimmunity: a nested case-control study among children with HLA-conferred susceptibility to type I diabetes. Eur J Clin Nutr 64, 792-799.

13. Virtanen SM, Nevalainen J, Kronberg-Kippila C, et al. (2012) Food consumption and advanced beta cell autoimmunity in young children with HLA-conferred susceptibility to type 1 diabetes: a nested case-control design. Am J Clin Nutr 95, 471-478.

14. Giacca A, Xiao C, Oprescu AI, et al. (2011) Lipid-induced pancreatic \{beta\}-cell dysfunction: focus on in vivo studies. Am J Physiol Endocrinol Metab 300, E255-E262.

15. Morgan NG \& Dhayal S (2010) Unsaturated fatty acids as cytoprotective agents in the pancreatic beta-cell. Prostaglandins Leukot Essent Fatty Acids 82, 231-236.

16. Kupila A, Muona P, Simell T, et al. (2001) Feasibility of genetic and immunological prediction of type I diabetes in a population-based birth cohort. Diabetologia 44, 290-297.

17. Erkkola M, Karppinen M, Javanainen J, et al. (2001) Validity and reproducibility of a food frequency questionnaire for pregnant Finnish women. Am J Epidemiol 154, 466-476.

18. Kukko M, Kimpimaki T, Korhonen S, et al. (2005) Dynamics of diabetes-associated autoantibodies in young children with human leukocyte antigen-conferred risk of type 1 diabetes recruited from the general population. J Clin Endocrinol Metab 90, 2712-2717.

19. Willett W (1998) Energy-adjusted or residual method. In Nutritional Epidemiology, pp. 288-291. New York, NY: Oxford University Press.

20. Siljander HT, Simell S, Hekkala A, et al. (2009) Predictive characteristics of diabetes-associated autoantibodies among children with HLA-conferred disease susceptibility in the general population. Diabetes 58, 2835-2842.

21. Erkkola M, Kronberg-Kippila C, Savilahti E, et al. (2005) Maternal consumption of dairy products during pregnancy and lactation, and the development of cow's milk antibodies in the offspring. Acta Paediatr 94, 696-704.

22. Luopajarvi K, Savilahti E, Virtanen SM, et al. (2008) Enhanced levels of cow's milk antibodies in infancy in children who develop type 1 diabetes later in childhood. Pediatr Diabetes 9, 434-441.

23. Knip M, Virtanen SM \& Akerblom HK (2010) Infant feeding and the risk of type 1 diabetes. Am J Clin Nutr 91, 1506S-1513S.

24. Knip M, Virtanen SM, Seppa K, et al. (2010) Dietary intervention in infancy and later signs of beta-cell autoimmunity. N Engl J Med 363, 1900-1908.

25. Savilahti E \& Saarinen KM (2009) Early infant feeding and type 1 diabetes. Eur J Nutr 48, 243-249.

26. Galli C \& Calder PC (2009) Effects of fat and fatty acid intake on inflammatory and immune responses: a critical review. Ann Nutr Metab 55, 123-139.

27. Virtanen SM, Uusitalo L, Kenward MG, et al. (2011) Maternal food consumption during pregnancy and risk of advanced beta-cell autoimmunity in the offspring. Pediatr Diabetes $\mathbf{1 2}$ 95-99.

28. Nwaru BI, Erkkola M, Lumia M, et al. (2012) Maternal intake of fatty acids during pregnancy and allergies in the offspring. Br J Nutr Nov 108, 720-732.

29. Lumia M, Luukkainen P, Tapanainen H, et al. (2011) Dietary fatty acid composition during pregnancy and the risk of asthma in the offspring. Pediatr Allergy Immunol 22, 827-835. 